rheuma plus $2019 \cdot 18: 191$

https://doi.org/10.1007/s12688-019-00295-5

(c) Springer-Verlag GmbH Austria, ein Teil von Springer Nature 2019

\title{
Doz. Dr. Attila Dunky verstorben
}

Liebe Leserinnen und Leser,

mich hat die Nachricht vom unerwarteten Ableben Attila Dunky's aus zweierlei Gründen sehr betroffen gemacht. Zum einen stand er nach wie vor mitten in seinem rheumatologischen Wirken und Attila Dunky lag die Rheumatologie im wahrsten Sinne des Wortes am Herzen. Zum zweiten verliere ich mit ihm den zweiten und letzten meiner beiden rheumatologischen Mentoren, nach Rudi Eberl. Beide standen für eine patientenorientierte Rheumatologie des Behandelns im wahrsten Sinne des Wortes, nämlich Hand anzulegen an den Patienten und einzugehen auf individuelle Probleme.

Attila Dunky fungierte von 2002 bis 2004 als Präsident der Österreichischen Gesellschaft für Rheumatologie. Er war im Besitze zahlreicher Auszeichnungen und Ehrungen, was Zeugnis ablegt für das hohe Ansehen, das er in Fachkreisen, aber auch darüber hinaus, genoss.

Attila Dunky, mit dem ich über ein Dezennium die D-Station der II. Medizinischen Abteilung im KH Lainz betreuen durfte und dem ich auch schließlich als stationsführender Oberarzt nachgefolgt bin, hat mich persönlich sehr Vieles gelehrt. Attila war ein Meister der Nadel, denn der intraartikulären Injektion galt sein besonderes Interesse. Die Krönung seiner beruflichen Laufbahn war die Berufung an die Spitze der V. Medizinischen Abteilung des WSP, der er seine ganze Energie in der Folge widmete. Daneben galt seiner Ordination, die er bis zuletzt in vollem Umfang betrieb, sein Hauptaugenmerk; dort war er das, was er am liebsten war, einfach Arzt.

Nicht zuletzt fungierte er seit Gründung dieser Zeitschrift als deren Herausgeber, bis er sich im Jahre 2011 auf die Position des Senior Editors zurückzog, ohne aber den Weg von rheuma plus je aus den Augen zu

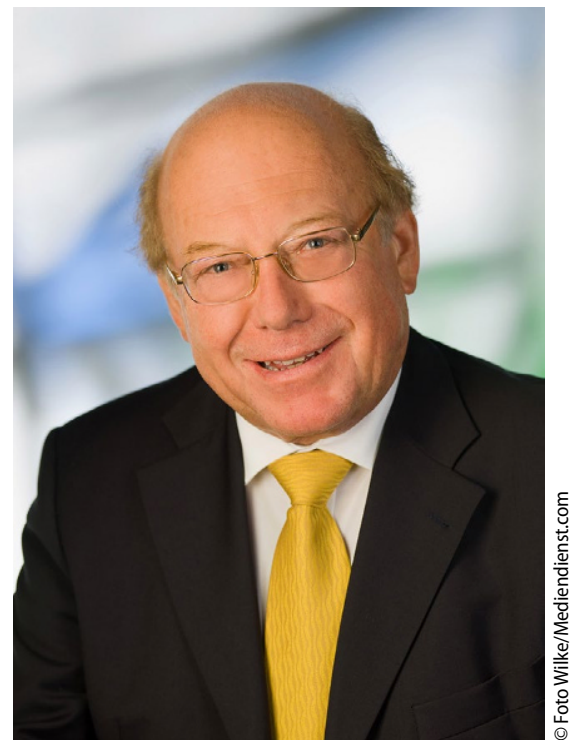

$\Delta$ Doz. Dr. Attila Dunky

lassen. Ebenfalls von Beginn an unterstützte er tatkräftig das Österreichische BiologikaRegister BioReg.

Attila Dunky war aber auch ein äußerst kommunikativer und geselliger Mensch, der abseits der Rheumatologie viele Interessen verfolgte. Die vielen Abende in geselliger und launiger Runde werde ich nie vergessen. Leider ist es nicht mehr dazu gekommen, dass ich mit ihm gesegelt (seine große Leidenschaft) wäre.

Wir alle verlieren mit Attila Dunky einen Freund, einen Kollegen mit großer Kompetenz und einen großen Humanisten. Ich werde nie vergessen, dass Attila mein Leben deutlich geprägt hat, und bin dankbar dafür, dass ich mich zu seinen Freunden zählen durfte. Unsere Gedanken sind jetzt bei seiner Familie.

Lieber Attila, die Erde werde Dir leicht.

Burkhard Leeb 